\title{
A movie of the RNA polymerase nucleotide addition cycle Florian Brueckner ${ }^{1,3}$, Julio Ortiz ${ }^{2}$ and Patrick Cramer ${ }^{1}$
}

During gene transcription, RNA polymerase (Pol) passes through repetitive cycles of adding a nucleotide to the growing mRNA chain. Here we obtained a movie of the nucleotide addition cycle by combining structural information on different functional states of the Pol II elongation complex (EC). The movie illustrates the two-step loading of the nucleoside triphosphate (NTP) substrate, closure of the active site for catalytic nucleotide incorporation, and the presumed two-step translocation of DNA and RNA, which is accompanied by coordinated conformational changes in the polymerase bridge helix and trigger loop. The movie facilitates teaching and a mechanistic analysis of transcription and can be downloaded from http://www.Imb.uni-muenchen.de/cramer/pr-materials.

\footnotetext{
Addresses

${ }^{1}$ Gene Center and Center for Integrated Protein Science Munich (CIPSM), Department of Chemistry and Biochemistry, LudwigMaximilians-Universität München, Feodor-Lynen-Strasse 25, 81377 Munich, Germany

${ }^{2}$ Max Planck Institute of Biochemistry, Department of Molecular Structural Biology, Am Klopferspitz 18, 82152 Martinsried, Germany

Corresponding author: Cramer, Patrick (cramer@Imb.uni-muenchen.de) ${ }^{3}$ Present address: Membrane Protein Laboratory, Imperial College London, Diamond Light Source Ltd, Harwell Science and Innovation Campus, Didcot, OX11 ODE, United Kingdom.
}

Current Opinion in Structural Biology 2009, 19:294-299

This review comes from a themed issue on Nucleic acids

Edited by Eric Westhof and Dinshaw J Patel

Available online 27th May 2009

0959-440X/\$ - see front matter

(C) 2009 Elsevier Ltd. All rights reserved.

DOI 10.1016/j.sbi.2009.04.005

\section{Introduction}

In all cells, gene transcription is carried out by multisubunit RNA polymerases that share a highly conserved active center. During RNA elongation, the polymerase moves along a DNA template and synthesizes a complementary RNA chain. Extension of the RNA chain is achieved during the nucleotide addition cycle (NAC). The NAC begins with binding of a nucleoside triphosphate (NTP) substrate to the transcription elongation complex (EG) that is formed by the polymerase, DNA, and RNA. Catalytic addition of the nucleotide to the growing 3 -end of the RNA leads to formation of a pyrophosphate ion. Pyrophosphate release leads to the pre-translocation state, in which the newly added, $3^{\prime}$-terminal RNA nucleotide remains in the substrate site.
Finally, translocation of DNA and RNA results in the post-translocation state, in which the substrate site is free for binding the next NTP, and the NAC can be repeated. Recent structural studies of ECs of the eukaryotic Pol II (RNA polymerase II) and the bacterial RNA polymerase have revealed different functional states that reflect intermediates in the NAC. Here we combined available structures into a movie of the NAC that we provide to the community.

\section{EC structures}

The EC structure was originally modeled for the bacterial enzyme [1], and determined by X-ray crystallography for eukaryotic Pol II [2-4]. These studies revealed that DNA enters a central cleft of the enzyme. At the floor of the cleft, the active center harbors a hybrid duplex formed between the DNA template strand and the RNA product. The NTP substrate was trapped in the insertion site $\left[5,6^{\bullet \bullet}\right]$, which is formed by closure of the active site and apparently occupied during catalysis, but also in an overlapping site within an open active center, corresponding to a pre-insertion state [3]. Recent crystallographic structures of a bacterial EC showed a conserved arrangement of DNA and RNA [7], and revealed binding of the NTP in the insertion site, and in the pre-insertion state $\left[8^{\bullet \bullet}\right]$. NTP binding to the insertion site leads to a complete folding of the trigger loop, a mobile part of the active center $\left[6^{\bullet \bullet}\right]$. The NTP binds two catalytic metal ions $[5,9]$, consistent with a general two-metal ion mechanism for all polymerases [10]. Metal ion A is persistently bound to the active site, whereas metal ion B binds to the NTP triphosphate and apparently enters with the NTP. In the pre-insertion state, the NTP triphosphate and metal B are too far from metal A to permit catalysis.

\section{Two-step mechanism of substrate loading}

Available structural information on RNA polymerases and their ECs suggests a conserved NAC mechanism in all three kingdoms of life (Figure 1). The NTP first adopts the pre-insertion state and binds to an open active center conformation $\left[3,8^{\bullet \bullet}\right]$. Folding of the trigger loop then leads to closure of the active center, delivery of the NTP to the insertion site, and formation of all contacts required for catalysis $\left[6^{\bullet \bullet}, 8^{\bullet \bullet}\right]$. The two-step mechanism helps to explain selection of the correct NTP. NTP selection would begin by sampling of substrates in the pre-insertion state, where Watson-Crick interactions between the correct NTP and the DNA template are established. Additional contacts discriminate the NTP against desoxy-NTPs, to prevent synthesis of DNA. The correct NTP is then delivered to the insertion site, where it is double-checked. Subsequent catalysis leads to RNA 


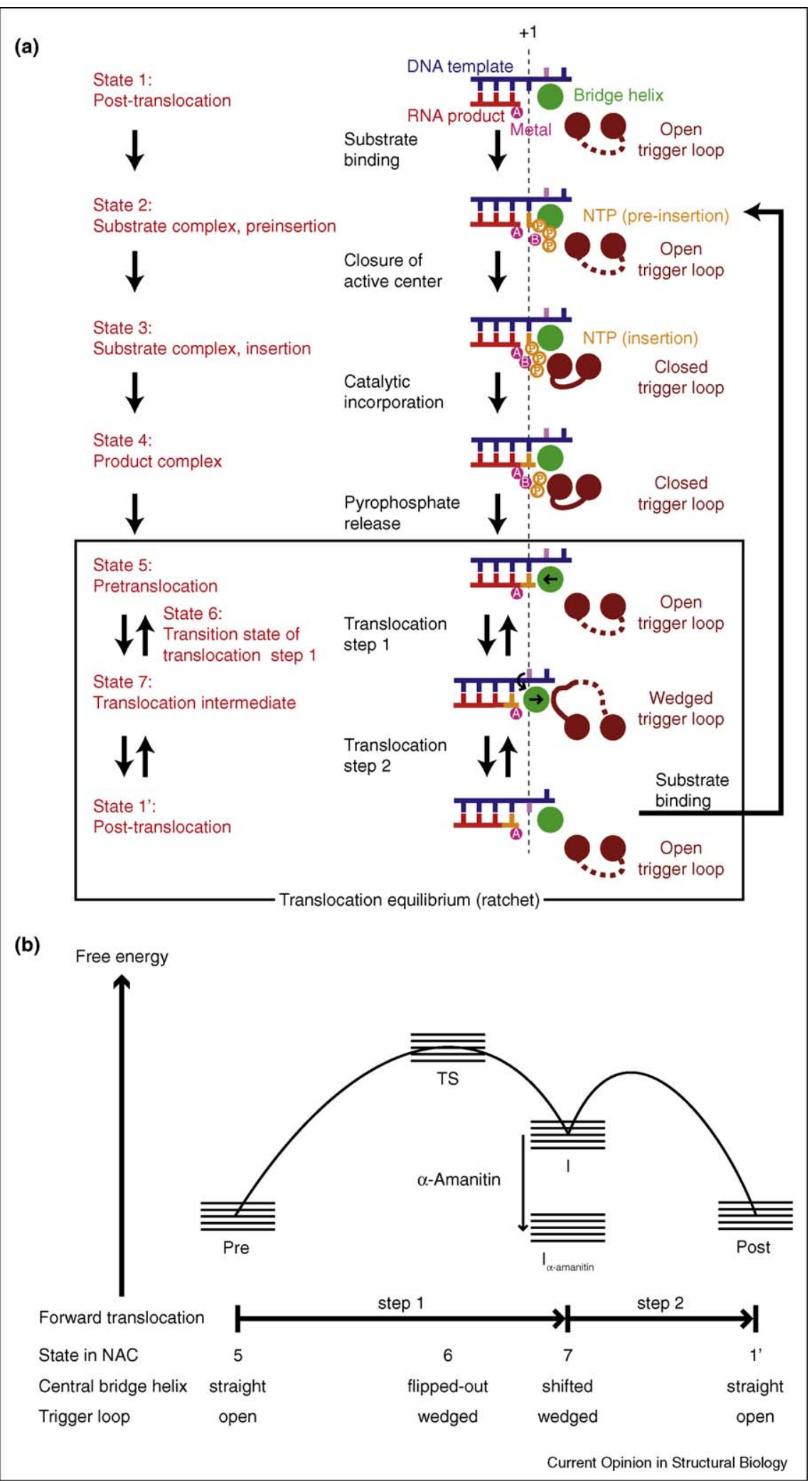

Model of the nucleotide addition cycle (NAC). (a) Schematic representation of the NAC. The seven NAC states are indicated on the left. The vertical

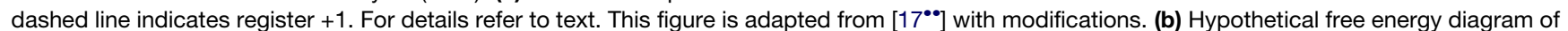
translocation. The energy level of the pre-translocation state (Pre) and the post-translocation state (Post) and the presumed intermediate probably depends on the nucleic acid sequence and other factors. The translocation pathway includes a translocation intermediate (I), which is apparently stabilized by $\alpha$-amanitin $\left(I_{\alpha \text {-amanitin }}\right)\left[17^{\circ}\right.$ ]. The translocation intermediate may be reached via a transition state (TS). 
extension and pyrophosphate formation. The structure of the resulting 'product complex' is unknown. It is, however, expected that release of pyrophosphate destabilizes the closed conformation of the active center, leading to trigger loop unfolding.

\section{Possible two-step mechanism of translocation}

A comparison of the free crystal structures of core Pol II and a bacterial RNA polymerase [11] initially suggested that translocation involves a conformational change in the active center bridge helix [2,9]. Bridge helix movement was suggested to be influenced by the adjacent trigger loop, which contacted the flipped-out, central region of the bridge helix in a structure of another bacterial RNA polymerase [12]. Functional cooperation of the bridge helix and trigger loop was revealed in biochemical studies of bacterial and archaeal RNA polymerases [13-15]. The functional relevance of bridge helix movement was supported by alternative bridge helix conformations observed within the same polymerase [16].

A recent structure of the Pol II EC bound by the inhibitor $\alpha$-amanitin trapped the trigger loop in a 'wedged' conformation, and stabilized the EC in a previously unobserved state that was intermediary between the pretranslocation and post-translocation states and apparently corresponded to a translocation intermediate $\left[17^{\bullet \bullet}\right]$. The next templating DNA base adopted a pre-templating position outside the active site above the bridge helix, and the trigger loop adopted a position where its residue L1081 formed a wedge that apparently stabilized the shifted central bridge helix. The trigger loop residue corresponding to L1081 in the bacterial RNA polymerase (M1238 in T. thermophilus) adopts a similar position in structures of the T. thermophilus (Tth) RNA polymerase holoenzyme [12] and the T. aquaticus (Taq) RNA polymerase bound to antibiotics [18]. In these structures, the central bridge helix adopts a flipped-out conformation in the region that is shifted in the Pol II EC intermediate.

These data suggested a conserved two-step mechanism of translocation via a trigger loop-stabilized EC intermediate with an altered structure of the central bridge helix [17 ${ }^{\bullet \bullet}$ ] (Figure 1a). During step 1, the hybrid moves from the pre-translocation to the post-translocation position, and the downstream DNA translocates until the next DNA template base (register +2 ) reaches the pre-templating position above the bridge helix. During step 2, the DNA template base twists by $90^{\circ}$, to reach its templating position in the active center (register +1 ). This twisting is accompanied by a flipping of the phosphate backbone group between the DNA template bases +1 and +2 , and a sliding of the downstream DNA to the post-translocation position. Modeling shows that the flipped-out bridge helix conformation can be accommodated within the Pol II EC intermediate structure $\left[17^{\bullet \bullet}\right]$. If the central bridge helix is only transiently flipped out, this EC conformation could represent the transition state of translocation step 1. A hypothetical free energy diagram for this two-step translocation mechanism is provided in Figure 1b.

\section{Design of the NAC movie}

We prepared two animations, an overview of the Pol II EC structure and the movie of the NAC. The first animation sets the direction of view of the active center that is then used in the second animation, the movie of the NAC. The first animation shows the rotating complete Pol II EC structure as a ribbon model with the twelve different polymerase subunits and the three nucleic acid strands in different colors. It then shows the nucleic acids as backbone models on a semitransparent molecular surface of the polymerase from different points of view, looking into the active center cleft and into the pore underneath the active site. Finally, the animation zooms in the active center, and stops in the direction of view used in the NAC movie.

To prepare the NAC movie, we assumed seven different functional states of the EC (Figure 1). Five of these states have been trapped crystallographically as Pol II EC structures, states $1,2,3,5$, and 7 , which correspond to the post-translocation, pre-insertion, insertion, pre-translocation, and translocation intermediate states, respectively. Two other states had to be modeled. State 4 corresponds to the product complex that could be trapped for the unrelated T7 RNA polymerase [19] but not for any cellular RNA polymerase. State 6 , which corresponds to the presumed transition state during translocation step 1, was modeled with the use of a free bacterial RNA polymerase conformation. The movie was designed to begin and end with the post-translocation state, such that repetitive viewing provides the impression of continuous RNA elongation by Pol II. The movie was further designed to visualize the coordinated movements of the central bridge helix and trigger loop, and to highlight the large-scale swinging of residue L1081 of the trigger loop between functional states of the EC.

\section{Preparation of coordinate files}

Pol II was modeled with its structure in the complete Pol II post-translocation EC (PDB ID 1Y1W, Table 1), except for the bridge helix and trigger loop, which differ in different EC structures. Alternative bridge helix and trigger loop conformations were modeled by superimposing unaltered parts in different structures. A common nucleic acid sequence, which was compatible with all structures, was designed based on the nucleic acid scaffold in the post-translocation EC (Figure 2). The nucleic acids, substrate NTPs, product pyrophosphate and metal $\mathrm{B}$ were placed by superimposing residues that were unchanged in different structures with the use of the LSQ and SSM functions in the program Coot [20]. 
Table 1

Structures used to create the NAC movie.

\begin{tabular}{|c|c|c|c|c|}
\hline Structure & Polymerase & Used to model NAC state & PDB-code & Reference \\
\hline Post-translocation EC & Complete yeast Pol II & All & $1 \mathrm{Y} 1 \mathrm{~W}$ & [3] \\
\hline EC substrate complex, pre-insertion state & Complete yeast Pol II & 2 & $1 Y 77$ & [3] \\
\hline EC substrate complex, insertion state & Core yeast Pol II & 3,4 & $2 \mathrm{E} 2 \mathrm{H}$ & {$\left[6^{\circ} \cdot\right]$} \\
\hline Pre-translocation EC & Core yeast Pol II & 4,5 & $116 \mathrm{H}$ & [2] \\
\hline$\alpha$-Amanitin-inhibited EC & Complete yeast Pol II & 6,7 & 2VUM & {$\left[17^{\bullet \bullet}\right.$} \\
\hline Free RNA polymerase & T. thermophilus holoenzyme & 6 & $1 \mathrm{IW} 7$ & [12] \\
\hline
\end{tabular}

To model state 1 , the sequence of the nucleic acids in structure $1 \mathrm{Y} 1 \mathrm{~W}$ was adjusted to the designed scaffold (Figure 2) and the downstream DNA was extrapolated as standard B-DNA from position +10 to position +15 . To model state 2, the NTP was placed at its observed position in the pre-insertion state in structure 1Y77 (Table 1). Metal $\mathrm{B}$ was placed to coordinate the triphosphate of the incoming NTP. To model state 3 , the NTP and the trigger loop residues 1065-1099 were modeled according to the structure of the Pol II EC with an NTP bound in the insertion site (2E2H, Table 1). To model state 4, the newly incorporated NMP at position +1 was placed according to the position of the $3^{\prime}$-nucleotide in the structure of the pretranslocation EC (1I6H, Table 1), the pyrophosphate was modeled assuming that it remains in the position of the beta-phosphate and gamma-phosphate of the NTP in the insertion site, and the trigger loop was assumed to remain closed (2E2H, Table 1). To model state 5 , the polymerase structure of the post-translocation EC (1Y1W, Table 1), including the open trigger loop, was combined with the RNA 3'-terminal nucleotide of the pre-translocation EC (1I6H, Table 1). To model state 6 , nucleic acids, trigger loop residues 1071-1101, and bridge helix residues 810 827 were taken from the $\alpha$-amanitin-inhibited EC struc-

Figure 2

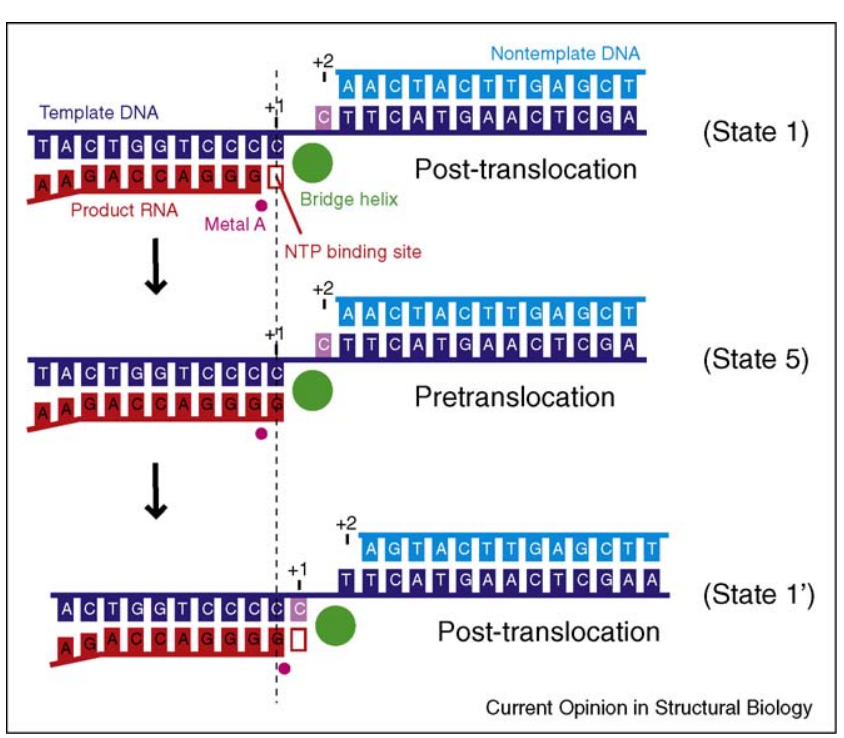

Nucleic acid sequences used in the NAC movie. ture (2VUM, Table 1, Figure 2). The flipped-out bridge helix residues 828-848 were taken from the T. thermophilus RNA polymerase structure (1IW7, Table 1). To model state 7, nucleic acids, trigger loop residues 1071-1102, and bridge helix residues $810-846$ were taken from the $\alpha$-amanitin-inhibited EC structure (2VUM, Table 1). The final state, state $1^{\prime}$ is identical to state 1 except that the RNA is extended by one nucleotide and the register of the nucleic acids has shifted. It was modeled by adjusting the sequence of the nucleic acids in the model for state 1 (Figure 2). These efforts resulted in eight sets of atomic coordinates corresponding to the seven states of the NAC and state $1^{\prime}$.

\section{Technical implementation}

The eight different coordinate sets were visualized and animated as follows. A Pol II density map was calculated from the protein part of the post-translocation EC structure $(1 \mathrm{Y} 1 \mathrm{~W}$, Table 1$)$ at a resolution of $0.5 \mathrm{~nm}$ in a volume of $(140)^{3}$ voxels using EMAN1 [21]. A corresponding surface was calculated and simplified in Amira (Visage Imaging GmbH, Germany). Regions from all structures listed in Table 1 were selected for animation. The morphing between each pair of consecutive conformations was performed in Chimera [22], using the interpolation method corkscrew with 20 interpolation steps between each state. The coordinates for each interpolated model were imported into the software $3 \mathrm{ds}$ Max (Autodesk, USA), allowing additional linear interpolation to achieve 60 frames between states. A ball and stick representation equivalent to that implemented in Chimera was chosen for nucleic acids and residue L1081 of the trigger loop. A simple tubular representation was used for the trigger loop and the bridge helix. The rendering of single frames was performed in $3 \mathrm{ds}$ Max and additional editing was done with Adobe Premiere Pro 2.0 (Adobe Systems Inc, USA). Snapshots of the different states of the EC taken from the NAC movie are shown in Figure 3.

\section{Limitations and future directions}

The obtained movie of the NAC illustrates our current mechanistic understanding of the NAC as an ordered, stepwise process. It does not visualize the stochastic thermal fluctuations of the EC that probably underlie sampling of the different NTP substrates, and the dynamic Brownian ratchet that probably underlies EC 
Figure 3

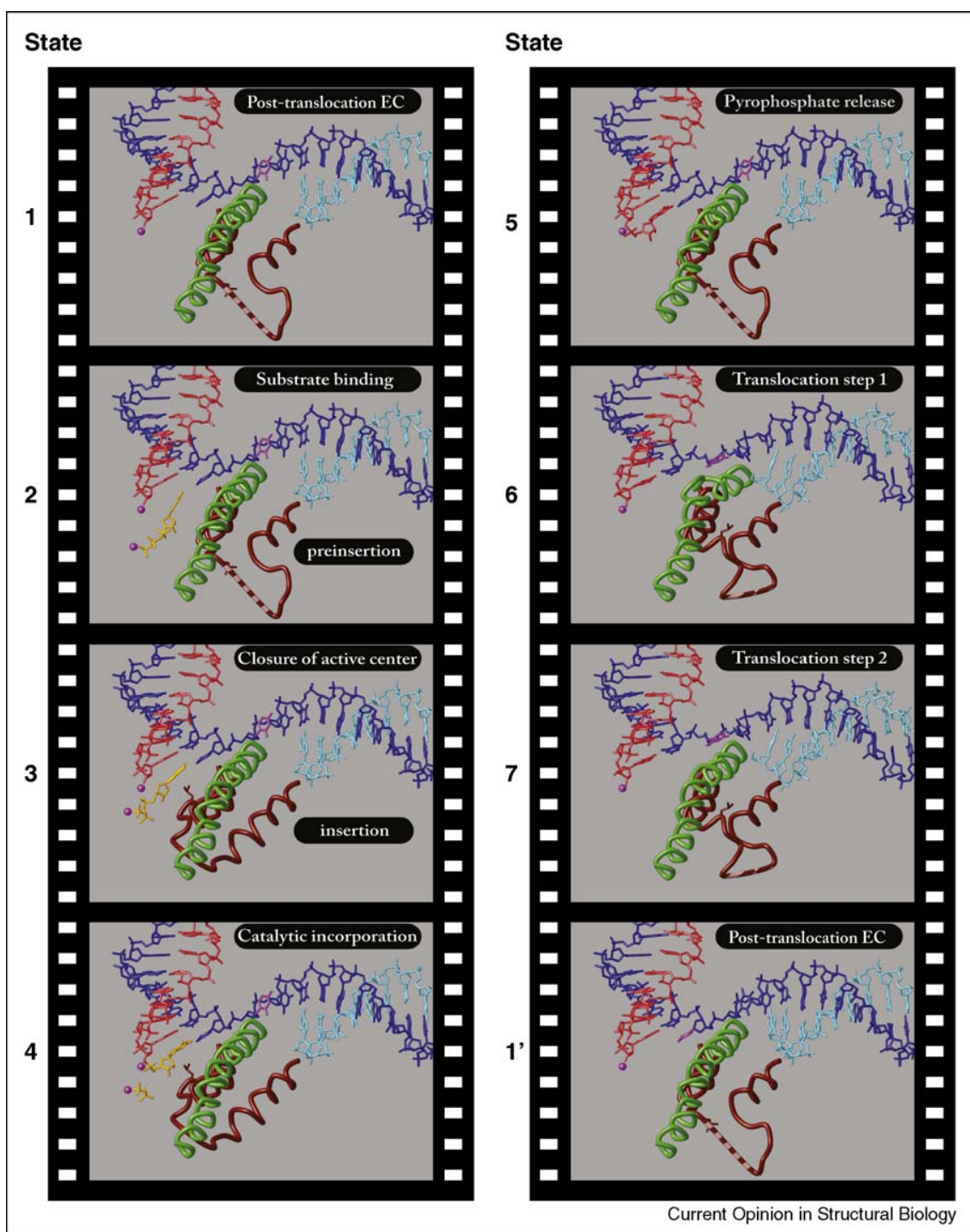

Snapshots from the NAC movie. Representative still images from the movie have been depicted that correspond to different functional states of the EC as shown in Figure 1. For details refer to text.

oscillation between pre-translocation and post-translocation states and directional polymerase movement $([14,23,24]$ and references therein). The Brownian ratchet model assumes that the ground state of the EC is an equilibrium between inter-converting pre-translocation and post-translocation states (the ratchet). NTP binding temporarily stops the oscillation, acting like a pawl of a ratchet. After nucleotide incorporation, oscillation resumes around the next template position. In this model, the energy for translocation comes from Brownian fluctuations, and the directionality of translocation results from trapping the forward fluctuation by NTP binding and nucleotide incorporation. Swinging of the trigger loop between closed and wedged positions, and the accom- panying shifting of the central bridge helix, are apparently important for the NAC and the Brownian ratchet.

In the future, the movie will probably be modified when additional structures and biochemical data become available. Future studies of the NAC should attempt to structurally trap additional states of the EC that may exist, to measure the fast kinetic parameters for the NAC, to simulate parts of the NAC by molecular dynamics, and to dissect the molecular basis of off-line events such as pausing, misincorporation, and proofreading. In the meantime, the derived movie of the NAC can be used to teach our current understanding of the molecular basis of the fundamental transcription mechanism. 


\section{References and recommended reading}

Papers of particular interest published within the period of review have been highlighted as:

- of special interest

$\bullet$ of outstanding interest

1. Korzheva N, Mustaev A, Kozlov M, Malhotra A, Nikiforov V, Goldfarb A, Darst SA: A structural model of transcription elongation. Science 2000, 289:619-625.

2. Gnatt AL, Cramer P, Fu J, Bushnell DA, Kornberg RD: Structural basis of transcription: an RNA polymerase II elongation complex at 3.3 A resolution. Science 2001, 292:1876-1882.

3. Kettenberger H, Armache K-J, Cramer P: Complete RNA polymerase II elongation complex structure and its interactions with NTP and TFIIS. Mol Cell 2004, 16:955-965.

4. Westover KD, Bushnell DA, Kornberg RD: Structural basis of transcription: separation of RNA from DNA by RNA polymerase II. Science 2004, 303:1014-1016.

5. Westover KD, Bushnell DA, Kornberg RD: Structural basis of transcription: nucleotide selection by rotation in the RNA polymerase II active center. Cell 2004, 119:481-489.

6. Wang D, Bushnell DA, Westover KD, Kaplan CD, Kornberg RD:

-. Structural basis of transcription: role of the trigger loop in substrate specificity and catalysis. Cell 2006, 127: 941-954.

The structure of a core yeast Pol II EC with bound NTP substrate provides a view of the insertion state and reveals a closed trigger loop that stacks against the substrate.

7. Vassylyev DG, Vassylyeva MN, Perederina A, Tahirov TH, Artsimovitch I: Structural basis for transcription elongation by bacterial RNA polymerase. Nature 2007, 448:157-162.

8. Vassylyev DG, Vassylyeva MN, Zhang J, Palangat M,

- Artsimovitch I, Landick R: Structural basis for substrate loading in bacterial RNA polymerase. Nature 2007, 448:163-168.

Structures of the bacterial EC bound to NTP analogs are reported in the presence and in the absence of the antibiotic streptolydigin. In the absence of the antibiotic, NTP binds to the insertion site and the trigger loop is closed. In the presence of the antibiotic, NTP binds in an overlapping site of the pre-insertion state and the trigger loop is partially open. Together with biochemical data, the results support the two-step mechanism of substrate loading.

9. Cramer P, Bushnell DA, Kornberg RD: Structural basis of transcription: RNA polymerase II at $\mathbf{2 . 8}$ angstrom resolution. Science 2001, 292:1863-1876.

10. Steitz TA: A mechanism for all polymerases [news; comment]. Nature 1998, 391:231-232.

11. Zhang G, Campbell EA, Minakhin L, Richter C, Severinov K, Darst SA: Crystal structure of Thermus aquaticus core RNA polymerase at 3.3 A resolution. Cell 1999, 98:811-824.
12. Vassylyev DG, Sekine S, Laptenko O, Lee J, Vassylyeva MN, Borukhov S, Yokoyama S: Crystal structure of a bacterial RNA polymerase holoenzyme at 2.6 A resolution. Nature 2002, 417:712-719.

13. Epshtein V, Mustaev A, Markovtsov V, Bereshchenko O, Nikiforov V, Goldfarb A: Swing-gate model of nucleotide entry into the RNA polymerase active center. Mol Cell 2002, 10:623-634.

14. Bar-Nahum G, Epshtein V, Ruckenstein AE, Rafikov R, Mustaev A, Nudler $\mathrm{E}:$ A ratchet mechanism of transcription elongation and its control. Cell 2005, 120:183-193.

15. Tan L, Wiesler S, Trzaska D, Carney HC, Weinzierl RO: Bridge helix and trigger loop perturbations generate superactive RNA polymerases. $\mathrm{J} \mathrm{Biol} \mathrm{2008,} \mathrm{7:40.}$

16. Tuske S, Sarafianos SG, Wang X, Hudson B, Sineva E, Mukhopadhyay J, Birktoft JJ, Leroy O, Ismail S, Clark AD Jr et al.: Inhibition of bacterial RNA polymerase by streptolydigin: stabilization of a straight-bridge-helix active-center conformation. Cell 2005, 122:541-552.

17. Brueckner F, Cramer P: Structural basis of transcription

- inhibition by alpha-amanitin and implications for RNA polymerase II translocation. Nat Struct Mol Biol 2008, 15:811-818. Crystals are reported that apparently preserve the equilibrium of the pretranslocation and post-translocation states of the complete yeast Pol II EC. Soaking of the crystals with the mushroom toxin a-amanitin trapped the EC in a previously unobserved state that is intermediary between the pre-translocation and the post-translocation states. The next DNA template base is seen in a pretemplating position above the bridge helix. The central bridge helix is shifted and apparently stabilized by the trigger loop, which forms a wedge below the bridge helix.

18. Campbell EA, Pavlova O, Zenkin N, Leon F, Irschik H, Jansen R, Severinov K, Darst SA: Structural, functional, and genetic analysis of sorangicin inhibition of bacterial RNA polymerase. $E M B O J$ J 2005, 24:674-682.

19. Yin YW, Steitz TA: The structural mechanism of translocation and helicase activity in t7 RNA polymerase. Cell 2004, 116:393404.

20. Emsley P, Cowtan K: Coot: model-building tools for molecular graphics. Acta Crystallogr D Biol Crystallogr 2004, 60:2126-2132.

21. Ludtke SJ, Baldwin PR, Chiu W: EMAN: semiautomated software for high-resolution single-particle reconstructions. J Struct Biol 1999, 128:82-97.

22. Pettersen EF, Goddard TD, Huang CC, Couch GS, Greenblatt DM, Meng EC, Ferrin TE: UCSF Chimera-a visualization system for exploratory research and analysis. J Comput Chem 2004, 25:1605-1612.

23. Sousa R: Machinations of a maxwellian demon. Cell 2005, 120:155-156.

24. Landick R: Active-site dynamics in RNA polymerases. Cell 2004, 116:351-353. 